\title{
Etapas del método estadístico
}

\author{
Phases of the statistical method \\ Rosa Burgos-Martínez ${ }^{b}$, Virginia Argüelles-Pascual $^{a}$, Raúl H. Palacios ${ }^{c}$
}

\begin{abstract}
:
In order to carry out an investigation based on quantitative information and in this way obtain reliable results to the reality of the study. In this work a graphic scheme of the stages or phases that are carried put in the statistical method is presented. The stages include the following: collection, count, presentation, synthesis and finaly the analysis stage.
\end{abstract}

Keywords:

statistical method, phases, data collection, synthesis, analysis

\section{Resumen:}

Con el fin de realizar una investigación basada en información cuantitativa y de esta manera obtener resultados fiables a la realidad de estudio. En este trabajo se presenta un esquema gráfico de las etapas o fases que se realizan en el método estadístico. Las etapas comprende las siguientes: recolección, recuento, presentación, síntesis y finalmente la etapa de análisis.

\section{Palabras Clave:}

método estadístico, etapas, recolección de datos, síntesis, análisis

\section{Introducción}

En el método estadístico se realiza una serie de pasos con el fin de obtener resultados fiables del tema que se investiga. Esto es importante realizar para evitar obtener conclusiones erróneas. En este trabajo, se presenta una descripción gráfica de las etapas del método estadístico, las cuales son: recolección que se refiere a la medición de variables y recoge la información que se especifica en el diseño de la investigación, la recolección se puede realizar a través de la observación o complejos procedimientos de medición; recuento, en esta etapa la información recogida se somete a revision, clasificación y posteriormente a procesar en paquetes de software estadístico, en ocasiones el recuento se puede hacer de manera simple y en ocasiones require de computadoras o paquetes de software especiales para el manejo de datos; en la presentación se realizan cuadros o tablas y gráficos que permiten una visualización rápida y precisa de los datos obtenidos, en primer lugar, la elaboración de cuadros o tablas tiene como propósito de presenter los datos de manera que se pueda efectuar una revision numérica precisa de los mismos, y la elaboración de gráficos facilita la inspección rápida de la información; en la etapa de síntesis la información es resumida en forma de medidas que permiten expresar de manera sintética las principales propiedades numéricas de grandes series o agrupamientos de datos; y en la etapa de análisis se efectúa la comparación de las medidas de resumen previamente calculada a través de fórmulas estadísticas y el uso de tablas específicamente diseñadas, la etapa de análisis en concreto consiste en la comparación de los resultados.

\footnotetext{
a Autor de Correspondencia, Universidad Autónoma del Estado de Hidalgo, Email: apascual@uaeh.edu.mx

b Universidad Autónoma del Estado de Hidalgo, Email: bu415563@uaeh.edu.mx

c Universidad Autónoma del Estado de Hidalgo, Email: raul_palacios@uaeh.edu.mx
} 
Esta etapa es fundamental va que de ella depende que se dispongan de datos BACTOS Y CONFIABLES.

Puedes realuarse a veces por simple OESERVACOÓN Y Yen otras ocasiones se requere de complejos procedinientos de medición.

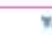

Consiste en la COMPARACION

Se efectuara la comparación de las medidas de resumen previamente caiculada

El uso de tablas especificamente diseriadas

Fórmulas estadísticas apropiadas

Esta etapa del método consiste en la MEDICOON DE VASABGLES
Tales procedinientos de muestreo están suborcinados a la consecuencia verficable que se desea comprobar $y$ al disefio de investigación seleccionado.

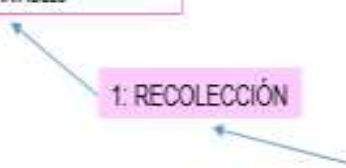

Para los datos cuantitativos, se encuentra la moda, amplitud, la medana, el promedio y la desviación estándar.

Permiten a los interlocutores evocar de la misma manera Ia ESENCIA DE LOS DATOS.

La información es resumida en forma de medidas cue permiten expresar de manera sintetica las principales prociedades numéricas de grandes series o agrupamientos de datos

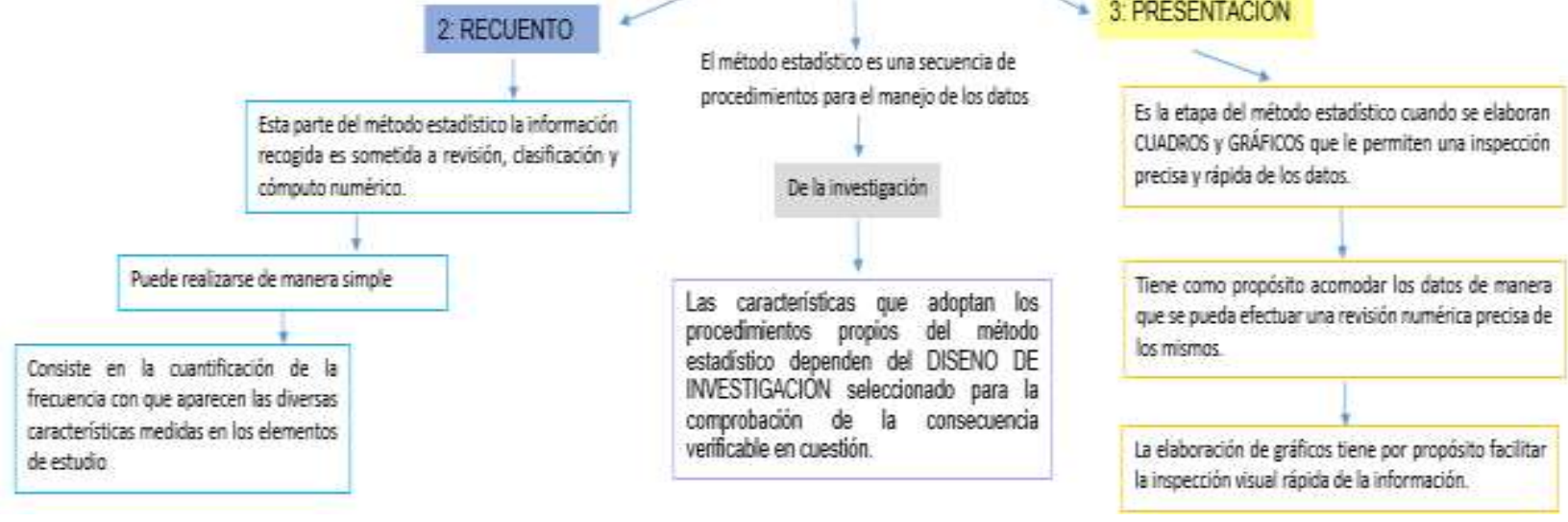

\section{Referencias}

[1] Imbaquingo D., Método estadístico.

[2] Sampieri, R. H., Collado, C. F., \& Lucio, P. B. (1991). Metodologia de la investigación.

[3] Bernal, C. A. (2010). Metodología de la investigación: administración, economía, humanidades y ciencias sociales. (3 Edic.). Colombia: Prentice Hall. 